\title{
P139 Does Early Life Programming Influence Arterial Stiffness and Central Hemodynamics in Adulthood? Could Birth Cohort Effects be of Importance?
}

\author{
Peter Nilsson*, Johannes Sperling \\ Lund University, Department of Clinical Sciences, Skane University Hospital, Malmo, Sweden
}

\section{ABSTRACT}

Objective: Our aim was to investigate possible associations between birth weight (BW) and adult carotid-femoral Pulse Wave Velocity (cfPWV) and augmentation pressure index (AIx).

Design: This study including 1598 participants, 340 elderly subjects and 1258 young-middle aged subjects from the Malmö Offspring Study with data on birth weight and gestational age (GA). Participants underwent cfPWV and AIx measurements with Sphygmocor (AtCor, Australia). Analysis of data was performed with multiple linear regression models with adjustments for age, sex, GA, and risk factors. Furthermore, comparisons were made between participants born prematurely or at term, or born small-for-gestational-age (SGA) or appropriate-for-gestational-age (AGA).

Results: Birth weight was positively associated with cfPWV after full adjustment $(b=0.057 ; p<0.001)$, Figure 1 , a finding that remained significant in the younger age group 18-27 years $(b=0.138, p=0.008)$. Furthermore, birth weight was inversely associated with AIx $(b=-0.058, p=0.001)$. Subjects born SGA had significantly higher AIx $(p=0.007)$ and MAP $(p=0.037)$ compared to AGA born. Preterm-born subjects showed significantly higher systolic blood pressure compared to term-born $(p=0.034)$. Also, BW was inversely associated with MAP $(b=-0.058, p=0.017)$ and SBP $(b=-0.047, p=0.031)$, respectively.

Conclusion: Our findings suggest that changes in total peripheral resistance, rather than decreased arterial stiffness, may explain the observed inverse association between birth weight and SBP. Furthermore, the association between birth weight and cfPWV, shown strongest in the youngest age group (birth cohort), could possibly be explained by increasing maternal overweight/obesity. This calls for screening of risk factors in subjects with adverse conditions at birth.

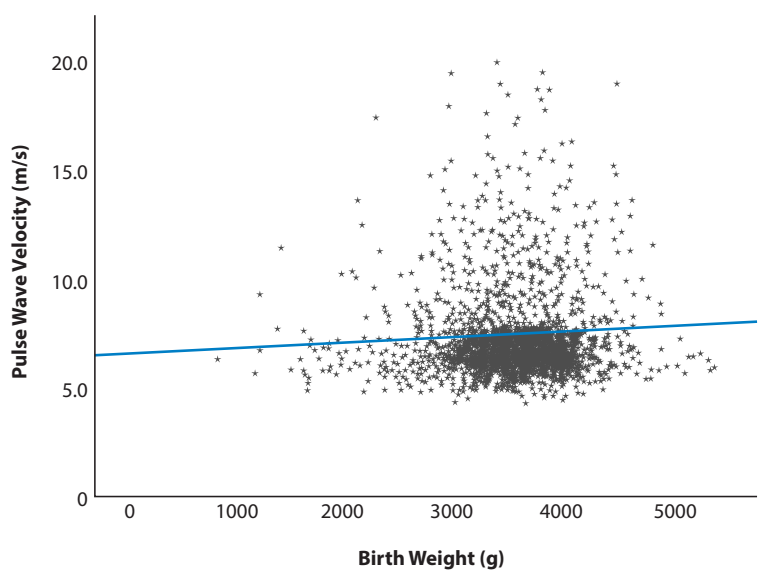

Figure $1 \mid$ Simple regression of birth weight and pulse wave velocity.

(c) 2019 Association for Research into Arterial Structure and Physiology. Publishing services by Atlantis Press International B.V. This is an open access article distributed under the CC BY-NC 4.0 license (http://creativecommons.org/licenses/by-nc/4.0/). 